\title{
RESEARCH ETHICS
}

\section{A public health perspective on research ethics}

\author{
D R Buchanan, F G Miller
}

J Med Ethics 2006;32:729-733. doi: 10.1136/jme.2006.015891

Ethical guidelines for conducting clinical trials have historically been based on a perceived therapeutic obligation to treat and benefit the patient-participants. The origins of this ethical framework can be traced to the Hippocratic oath originally written to guide doctors in caring for their patients, where the overriding moral obligation of doctors is strictly to do what is best for the individual patient, irrespective of other social considerations. In contrast, although medicine focuses on the health of the person, public health is concerned with the health of the entire population, and thus, public health ethics is founded on the societal responsibility to protect and promote the health of the population as a whole. From a public health perspective, research ethics should be guided by giving due consideration to the risks and benefits to society in addition to the individual research participants. On the basis of a duty to protect the population as a whole, a fiduciary obligation to realise the social value of the research and the moral responsibility to distribute the benefits and burdens of research fairly across society, how a public health perspective on research ethics results in fundamental re-assessments of the proper course of action for two salient topical issues in research ethics is shown: stopping trials early for reasons of efficacy and the conduct of research on less expensive yet less effective interventions.

See end of article for authors' affiliations

Correspondence to: D Buchanan, 306 Arnold House, School of Public Health and Health Sciences, University of Massachusetts, Amherst, MA 01003, USA;

Buchanan@schoolph. umass.edu

Received 3 January 2006 Revised 22 March 2006 Accepted 28 March 2006
$\mathrm{H}$ istorically, bioethics has drawn heavily on an ethical framework originally articulated to guide doctors in practising medicine, dating back to the Hippocratic oath: "I will follow that system or regimen which according to my ability and judgment I consider for the benefit of my patient and abstain from whatever is deleterious and mischievous." The Hippocratic oath affirmed that the primary moral obligation of the doctor is to benefit and not to harm the patient at hand. These principles had a major effect in framing the development of the field of bioethics. ${ }^{1}$ Yet, as Veatch ${ }^{2}$ notes, the Hippocratic oath is profoundly individualistic, proclaiming that the overriding moral obligation of doctors is strictly to do what is best for the individual patient, irrespective of other social considerations.

In recent years, there has been growing attention to ethics from a public health perspective. $^{3-5}$ Where medicine focuses on individual health, public health is concerned with the health of the entire population. Thus, in contrast with a fiduciary duty to the patient, public health ethics is founded on societal responsibility to protect and promote the health of the population as a whole. Our study examines the implications of acknowledging the moral obligation to protect population health in identifying the appropriate ethical norms to guide research ethics.

Health intervention research is conducted to achieve the goal of producing new knowledge. Such knowledge is valued because it is expected to improve healthcare services and the overall health of the population. Emanuel et $a l^{6}$ have posited that the first requirement necessary to make clinical research ethical is that it must be socially valuable. Research with no redeeming social value is unethical because it would not be possible to justify the risks of participation, and scarce resources would be wasted. If the primary purpose of conducting research is to realise socially valued goods, then one critical question rests on determining how the social value of health research should be assessed.

This analysis builds on a line of reasoning that has examined the role of justice in evaluating the conduct of clinical research. ${ }^{7}$ Many observers have noted a "paradigm shift" in the evolution of justice concerns in health research, moving from a predominant concern with protecting participants from harm to an emerging interest in improving access to clinical trials. ${ }^{8}$ In the historical context of public outrage at the egregious misconduct of researchers at Tuskegee and other research scandals, the Belmont Report ${ }^{9}$ concentrated accordingly on justice considerations internal to the research process. Although concerns about distributing the benefits of research fairly in society are presented, the report is largely framed around protecting research participants from harm and exploitation. Over time, however, the weight of public moral concerns has shifted, from a preoccupation with the risks of participating in research to a greater appreciation of the potential benefits. For example, the research community once thought that women should be excluded from health research due to a perceived greater vulnerability. Later, they recognised that it was unfair to conduct research only on men, as the benefits of the research for women would remain uncertain, and justice demands that the benefits be distributed fairly. ${ }^{10-12}$ Thus, the inclusion of various population groups is now seen to be obligatory, both to benefit potential participants and to expand medical knowledge about previously excluded or under-represented groups,

Abbreviations: KKI, Kennedy-Kreiger Institute; RCT, randomised controlled trial 
such as women, children and minorities. This paradigm shift opened the door to new claims on justice, expanding the range of parties that ought to be considered morally relevant beyond the participants alone in determining the value of the research under consideration.

In our paper, we argue that taking a public health perspective on research ethics is associated with broadening the conceptualisation of risks and benefits deemed ethically relevant in deliberations on health research. To ascertain its social value, a comprehensive analysis must take into account not only the risks and benefits to the research participants themselves but also the benefits and risks to the population as a whole. Many inferences can be drawn from this position. Here, we examine two topical issues where adopting a broader public health perspective results in fundamental reassessments of the proper course of action: (1) defining early stopping guidelines for clinical trials based on evidence of benefit and (2) permitting research on less expensive yet less effective interventions designed to promote the health of disadvantaged populations.

\section{PUBLIC HEALTH PERSPECTIVE ON TOPICAL RESEARCH CONCERNS \\ Early stopping for efficacy}

Randomised controlled trials (RCTs) constitute the most rigorous method for evaluating the safety and efficacy of novel health interventions. Ethical conduct of RCTs requires data monitoring during the trial to determine whether the research should be terminated earlier than planned. Stopping trials early based on adverse side effects is ethically unproblematic, but the question of when to stop trials based on emerging evidence of efficacy is more complex.

Similar to Fried' $\mathrm{s}^{13}$ original articulation of the concept of equipoise, bioethicists and medical researchers have claimed that clinical trials are ethical only if a state of uncertainty exists about whether a new treatment is superior to a comparison condition (typically, the current standard of care). In this perspective, trials must be stopped as soon as sufficient evidence emerges that the experimental intervention provides greater benefit, thus disturbing the state of equipoise. Marquis ${ }^{14}$ coined the phrase "therapeutic obligation" to denote the fundamental ethical norm to be upheld in this view of conducting randomised trials. On the basis of the professed therapeutic obligation to treat and benefit patientparticipants, he claimed that it is "ethically mandatory" to stop a trial when "the hypothesis that therapy A is inferior to therapy B is more probable than its opposite" (p 4l-2).

Although lengthy debates have ensued on what constitutes sufficient evidence to confirm a hypothesis in this context, a working consensus has emerged that a priori early stopping rules, such as the O'Brien-Fleming boundary, provide an acceptable basis for deciding that the apparent benefit is real and not a statistical anomaly. ${ }^{15}$ It is important to note that such early stopping rules are based on strict extrapolations of the standard $\mathrm{p}<0.05$ convention for determining significance at the end of a trial, appropriately adjusted to take into account well-known statistical problems that result from looking at the data several times. ${ }^{16}$ Once a stopping boundary has been crossed, the prevailing position is that it is morally imperative to decide whether to stop the trial to cease providing participants with an evidently inferior treatment.

Many problems, however, are associated with early stopping. Early stopping may leave doctors and patients floundering regarding the proper dose and length of treatment for the new drug. It may necessitate conducting additional trials to address questions left unanswered as a result of early stopping, exposing even more participants to the risks of research. It may force other trials to be stopped, as the standard of care gets recalibrated, thus resulting in even more information being lost outside of the trial in question. With relatively few outcome events (eg, death and disease recurrence), early stopping may lead to inflated estimates of the efficacy of the new intervention, thus providing false hope to future patients. ${ }^{17}$ Finally, it leaves everyone with greater uncertainty about the longer term benefits and risks of the treatment. ${ }^{18}$ On the basis of a perceived therapeutic obligation of doctors to offer optimal medical care, proponents of the current approach maintain that these are unavoidable costs essential for conducting research ethically. From a public health perspective, however, one critical concern is that stopping trials early based on emerging evidence of efficacy may result in a treatment that is ultimately found to have an unfavourable risk-benefit ratio. As the use of hormone replacement therapy and COX-2 inhibitors shows, a new treatment may provide short-term relief of symptoms, but cause higher mortality over the longer term. ${ }^{19}{ }^{20}$ Although it is generally not possible, for economic and pragmatic reasons, to conduct randomised trials for adequate duration to determine a definitive risk-benefit ratio, early stopping exacerbates the problems of insufficient data and uncertainty.

There are two major problems with the prevailing approach to stopping trials early for reasons of efficacy.

Firstly, in invoking the therapeutic obligation, the current approach misclassifies clinical research as a form of medical treatment. In contrast, if clinical research is characterised as scientific activity intended to produce new knowledge for social benefit, then it is necessary to identify ethical norms appropriate to the distinct context of conducting research. As clinical research uses methods unrelated to medical care (eg, randomisation and double blinding) and conducts procedures solely for data-gathering purposes that provide no benefit to participants (eg, biopsies and lumbar punctures), it is misleading to claim that norms developed for one type of social practice should be applied to another, different activity.

Secondly, from a public health perspective, the potential harm associated with withholding the perceived benefits from participants in the comparison arm must be balanced against the value of the knowledge to be gained about longerterm risks and benefits for the vastly larger population of future patients. Because randomised trials are scientific experiments designed to produce knowledge for improving healthcare, the risks and benefits to society as a whole must be considered to be morally valid. Therefore, we maintain that the ethically appropriate standard for stopping trials early for reasons of efficacy should be proof beyond a reasonable doubt that participants are truly being deprived of a clinically meaningful benefit. ${ }^{21}$ The reason why it is important to set a higher standard is to protect the large population of future patients from being exposed to a new treatment with an inadequately assessed risk-benefit profile. Although it is morally obligatory to protect the research participants from being exposed to undue risks of harm, a public health perspective requires a more stringent burden of proof to stop trials early and forgo further accumulation of valuable data than the early stopping rules now applied. ${ }^{17}$ Once we acknowledge the social purpose of health research, the appeal to the therapeutic obligation as an ethical framework to govern clinical trials must be regarded as inappropriate. From a public health perspective, the research community has a binding moral obligation to protect future patients as well as the current research participants.

A public health perspective on early stopping entails due recognition of the inherently social purpose of clinical research. In the context of providing medical care, doctors quite rightly have an unqualified duty of fidelity to each patient. However, in the context of conducting RCTs, the decisions of investigators, institutional review boards, and 
data and safety monitoring boards have implications for the whole of society. As regulatory approval and current standards of practice often hang in the balance, the moral consequences of being wrong, of recommending a treatment that may turn out to increase the risk of serious morbidities or death, are more severe than individual clinical decisions. It is therefore appropriate to require proof beyond a reasonable doubt of clinically important benefit to stop randomised trials early. It is socially irresponsible to hasten the use of new treatments without obtaining sufficiently rigorous data indicating that they offer a reasonable risk-benefit ratio. ${ }^{22}$ From a public health perspective, the decision to continue a trial is justified on grounds that it is in the public's interest to establish compelling evidence of the therapeutic merit of new treatments.

The position presented here acknowledges that early stopping may be ethically appropriate in carefully identified instances, despite the loss of valuable data. Well-defined limits must prohibit trials from continuing past the point of exploiting the research participants due to withholding better treatment, but because a public health framework is not derived from a perceived duty to benefit individual patientparticipants, early stopping guidelines based on the principles presented here are more conservative than currently existing stopping rules. By extension, we propose that when trials are stopped early, it is imperative that regulatory authorities require phase IV population monitoring to determine whether there are serious long-term side effects. As part of promulgating these new guidelines, it will be necessary to educate prospective participants about the differences between research and treatment, in general, and about interim data monitoring and the conditions under which trials will be stopped early, in particular, and to institute new processes of informed consent to clarify the protections provided.

\section{Research on less expensive yet less effective interventions}

As a result of the widespread conflation of conducting research with practising medicine, an analogous problem has arisen with respect to normative analyses of research on less expensive yet less effective treatment interventions. Invoking Kant's $^{23}{ }^{24}$ categorical imperative that people should never be treated merely as a means to an end, many analysts have argued that subjecting research participants to an experimental treatment regimen known to be inferior to existing alternatives is morally impermissible. In this view, it is considered exploitative to provide research participants with anything less than the best, because offering an inferior treatment would be sacrificing their welfare for the sake of science. Hence, heated charges of exploitation have been levelled at HIV perinatal transmission trials in developing countries, and likewise, at the Kennedy-Krieger Institute's (KKIs) Lead Paint Abatement Study. ${ }^{25}{ }^{26}$ We focus here on the second, but the principal ethical considerations also apply to the first.

In the KKI study, researchers set out to test less expensive lead abatement processes, which were known to be less than $100 \%$ effective, in the homes of disadvantaged inner-city residents. ${ }^{27}$ Critics claimed that this research was unethical because an effective, albeit expensive (roughly US\$20 000 per house), solution to the problem was known. In response, we contend that if complete lead abatement or the provision of lead-free housing is not likely to occur in the foreseeable future, then finding a less expensive means of partial abatement that can be implemented has social value. ${ }^{28}$ The value of this research lies in developing an intervention that can provide tangible relief to potentially millions of children. Although the technical capacity for complete lead abatement is available, the slow rate of progress in providing lead-free housing makes research aimed at finding more cost-effective methods to alleviate this serious public health problem a potentially positive contribution to improving the conditions of the least well off. Rather than standing on principles that call for far-reaching changes that are unlikely to occur in the near future, we argue that the failure to conduct such research causes the greater harm, because it limits health interventions to the status quo of those who can afford currently available options and deprives disadvantaged populations of imminent incremental improvements in health. Nevertheless, this research can be ethically justified only in carefully circumscribed conditions.

To justify public health research aimed at developing less expensive yet less effective interventions, five conditions must be met:

- a large population in need

- the existence of a more effective treatment standard that is substantially more expensive than a less costly intervention that is still hypothesised to be considerably effective

- economic or political constraints that do not allow universal provision of the higher standard

- a high degree of likelihood that the less expensive intervention will be implemented on a wide scale

- community endorsement of the research.

Under these conditions, research on less expensive, less effective interventions may be ethically warranted..$^{28}$ This position gives due moral consideration to the feasibility of providing universal public health protections with existing interventions.

Critics charge that the KKI participants were treated inequitably, ${ }^{25} 29{ }^{30}$ but the comparison is relative to better-off people who have access to new or refurbished housing. The indictment assumes that the best available treatment is owed unconditionally to all in need, and therefore, the feasibility of universal provision is ethically irrelevant. From a public health perspective, however, the feasibility of universal coverage is ethically valid and crucial to consider. If extant political economic conditions thwart the extension of the higher standard of care to the entire population, then the appropriate comparison group is to those who do not have access. Children in the KKI study were not being exposed to a home environment riskier than would otherwise be available to them as a result of their participation. On the contrary, the research was intended to alleviate the unjust living conditions of these children. Thus, the KKI study offered a favourable risk-benefit ratio both in terms of potential benefits to the participating children and in terms of the social value of knowledge to be gained. Research on less expensive, less effective interventions is justified by giving due moral consideration to the feasibility of providing population-wide protections, provided the risks to participants are reasonable and proportionately balanced in relation to the prospective health benefits to them and the value of the knowledge to be gained. Concerns about reducing pressure to provide the most effective intervention need to be assessed in the specific historical context of the health problem under consideration and to be balanced against the likelihood that the status quo of neglect will be maintained if a less expensive alternative is not developed.

Finally, from a public health perspective, it is important to recognise an ethical standard that respects community autonomy. ${ }^{31}$ This standard can be achieved by requiring community collaboration (ie, at a minimum, establishing a community advisory board) to protect against exploiting vulnerable populations, to ensure fair terms of cooperation, to ratify that the interventions to be tested are acceptable to 
community members, and to minimise potential misunderstandings about the research. Such community advisory boards should have responsibility for determining whether the research goals are valuable to local community members and the methods are acceptable before the research is allowed to proceed. To discuss concerns about situations in which the feasibility of providing a higher standard of care is contested, the community advisory board could decide whether to press the demand for the higher standard of care or allow the research to proceed. On the basis of the public health framework presented here, respect for communities entails a fundamental right of community members to exercise a meaningful role in determining the conduct of research that affects their lives.

\section{DISCUSSION}

The preceding analysis challenges the ethical framework currently governing the conduct of health research. The prevailing framework is based on a perceived therapeutic obligation to provide optimal medical care to people who participate in research, a duty derived from the Hippocratic oath to which doctors have long sworn their allegiance. In presenting an alternative public health perspective, we have disputed this perspective on three grounds: the duty to protect the population as a whole; a fiduciary obligation to realise the social value of the research; and the moral responsibility to distribute the benefits and burdens of research fairly across society.

Firstly, from a public health perspective, the research community has an overriding obligation to protect the entire population by developing adequate data about the safety of novel interventions before they are made publicly available. It is socially irresponsible to hasten new pharmaceutical products to market or validate new medical or surgical procedures if a conservative burden of proof has not been met and reasonable doubts persist about their therapeutic merit.

Secondly, it is essential to recognise that the purpose of conducting research is to produce new knowledge, knowledge that is valued because it leads to improvements in healthcare and in the health of the population as a whole. When research is cast as a form of medical care, the goal of realising the value of the research is undermined. Clearly, research participants must be protected from harm and exploitation, but their therapeutic needs must be seen in the context of volunteering to participate in research. Research inherently contains risks, but the participants should be willing to assume these risks, either because they are likely to be outweighed by the potential benefits to them or because their participation offers the prospect of benefit to the rest of society, with a very low likelihood of incurring serious harm.

Finally, it is unjust to discount the needs of the population as a whole in testing health interventions. It is unacceptable to focus exclusively on the participants alone, and not give due attention to the larger social ramifications of the research. Although the medical community has long argued that a fiduciary duty to the individual patient prohibits them from making medical decisions based on the cost of the treatment, this position is untenable in the context of conducting research aimed at improving healthcare and population health. From a public health perspective, population needs must be considered, and therefore the cost effectiveness of various treatment options taken as a valid moral concern. A concern for justice is ultimately about distributing social resources, rights and responsibilities fairly, especially in meeting the needs of the least well off. If large segments of the population are denied access to effective treatments because they cannot afford them, then justice has not been served. Justice demands that feasible solutions to health problems that disproportionately affect disadvantaged population groups be developed.

In conclusion, the prevailing approach to research ethics has failed to achieve a satisfactory balance between realising the value of research and protecting and benefiting participants. Rather than constructing an ethical framework based on norms appropriate to providing medical care, a public health perspective recognises the intrinsic social purpose of conducting research, and thus gives greater weight to the risks and benefits to society as a whole. In anticipation of possible accusations that we are merely promoting a crude utilitarian calculus that would inevitably lead to the abuse and exploitation of research participants, we must reiterate that both the research participants and the public have a vested interest in discovering safe and feasible remedies to important health problems. Giving greater weight to social considerations does not entail disregarding appropriate protections for individual participants. There can be little question that ethical norms that place appropriate constraints on pursuing societal benefit are essential to protect the rights and well-being of people. The potential for exploitation will continue to be constrained by the ethical imperatives of informed consent and monitoring by DSMBs. From a public health perspective, however, the critical issue is whether the risks and benefits of the research to society as a whole are given serious and appropriate consideration in these deliberations. We have presented the reasons why they should. In conclusion, a public health framework on research ethics is based on offering a fair social contract in which the risks to the participants are not disproportionate relative to the potential benefits, and the participants may choose whether to seek these benefits in exchange for their contribution to socially valuable knowledge.

\section{Authors' affiliations}

D R Buchanan, University of Massachusetts, Amherst, Massachusetts, USA

F G Miller, National Institutes of Health, Bethesda, Maryland, USA

Competing interests: None.

\section{REFERENCES}

1 Beauchamp T, Childress J. Principles of biomedical ethics, 5th edn. New York: Oxford University Press, 2001.

2 Veatch R. Disrupted dialogue: medical ethics and the collapse of physicianhumanist communication. New York: Oxford University Press, 2005

3 Childress JR, Faden R, Gaare L, et al. Public health ethics: mapping the terrain. J Law Med Ethics 2002;30:170-8.

4 O'Neill O. Public health or clinical ethics: thinking beyond borders. Ethics Int Affairs 2002; 16:35-45.

5 Kass N. An ethics framework for public health. Am J Public Health 2001;91:1776-82.

6 Emanuel E, Wendler D, Grady C. What makes clinical research ethical? JAMA 2000;283:2701-11.

7 McCarthy C. The evolving story of justice in Federal Research Policy. In: Kahn J, Mastroianni A, Sugarman J, eds. Beyond consent: seeking justice in research. New York: Oxford University Press, 1998:11-31.

8 Powers M. Theories of justice in the context of research. In: Kahn J, Mastroianni A, Sugarman J, eds. Beyond consent: seeking justice in research. New York: Oxford University Press, 1998: 147-65.

9 National Commission for the Protection of Human Subjects of Biomedical and Behavioral Research. The Belmont Report: ethical principles and guidelines for the protection of human subjects of research, Federal Register Document 79-12065. Washington, DC: Department of Health and Human Welfare, US Government Printing Office, 1979.

10 Dresser R. Wanted: single, white male for medical research. Hastings Cent Rep 1992;22:24-9.

11 Kahn J, Mastroianni A, Sugarman J. Changing claims about justice in research: an introduction and overview. In: Kahn J, Mastroianni A, Sugarman J, eds. Beyond consent: seeking justice in research. New York: Oxford University Press, 1998:1-25.

12 Weijer C, Crouch, RA. Why should we include women and minorities in randomized controlled trials? J Clin Ethics 1999;10:100-6.

13 Fried C. Medical experimentation: personal integrity and social policy. Amsterdam: North-Holland Publishing Company, 1974.

14 Marquis D. Leaving therapy to chance. Hastings Cent Rep 1983;13:40-7. 
$15 \mathbf{O}^{\prime}$ Brien P, Fleming T. A multiple testing procedure for clinical trials. Biometrics 1979;35:549-56.

16 Jennison C, Turnbull B. Group sequential methods with applications to clinical trials. Boca Raton: Chapman and Hall, 2000.

17 Buchanan D, Miller FG. Principles of early stopping of randomized trials for efficacy: a critique of equipoise and an alternative ethical framework. Kennedy Inst Ethics J 2005;15:163-80.

18 Montori VM, Devereaux PJ, Adhikari NK, et al. Randomized trials stopped early for benefit: a systematic review. JAMA 2005;294:2203-9.

19 Horton R. Vioxx, the implosion of Merck, and aftershocks at the FDA. Lancet 2004;364:1995-6.

20 Writing Group for the Women's Health Initiative Investigators. Risks and benefits of estrogen plus progestin in healthy postmenopausal women: principal results from the women's health initiative randomized controlled trial. JAMA 2002;288:321-33.

21 Pocock SJ. When (not) to stop a clinical trial. JAMA 2005;294:2228-30.

22 Gifford F. Community-equipoise and the ethics of randomized clinical trials. Bioethics 1995:9:127-48

23 Angell $M$. The ethics of clinical research in the third world. N Engl J Med 1997;337:847-9.
24 Angell M. Investigators' responsibilities for human subjects in developing countries. N Engl J Med 2000;342:967-9.

25 Spriggs M. Canaries in the mines: children, risk, non-therapeutic research, and justice. J Med Ethics 2004;30:176-81.

26 Farmer P, Campos NG. New malaise: bioethics and human rights in the global era. J Law Med Ethics 2004;32:243-51

27 Pollak J. The lead-based paint abatement repair and maintenance study in Baltimore: historic framework and study design. J Health Care Law Policy 2002;6:89-108.

28 Buchanan D, Miller FG. Justice and fairness in the Kennedy Krieger Institute Lead Paint Study: the ethical justification for public health research on less expensive yet less effective interventions. Am J Public Health 2006:96:15-22.

29 Pinder L. Commentary on the Kennedy Krieger Institute Lead Paint Repair and Maintenance Study. Neurotoxicol Teratol 2002;24:477-9.

30 Needleman $\mathrm{H}$. What is not found in the spreadsheets? Neurotoxicol Teratol 2002;24:459-61.

31 Emanuel EJ, Wendler D, Killen J, et al. What makes clinical research in developing countries ethical? The benchmarks of ethical research. J Infect Dis 2004; 189:930-7.

\section{BNF for Children 2006, second annual edition}

In a single resource:

- guidance on drug management of common childhood conditions

- hands-on information on prescribing, monitoring and administering medicines to children

- comprehensive guidance covering neonates to adolescents

For more information please go to bnfc.org 